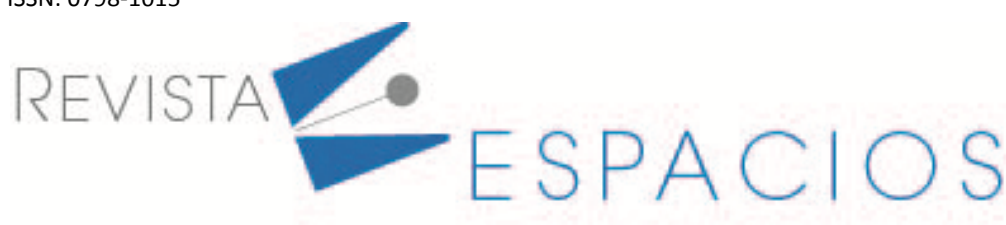

\title{
Proyecto arquitectónico como laboratorio vivo de investigación
}

\section{Architectural project as living research laboratories}

\author{
CARABAJO, Saturnino ${ }^{1}$ \\ TORRES, Rommy ${ }^{2}$ \\ REYES PINCAY, Brick ${ }^{3}$
}

\begin{abstract}
Resumen
El experimento se inició con la utilización de un entorno de aprendizaje denominado living labs, que son entornos de investigación de aprendizaje colaborativo para involucrar a los usuarios en la innovación y el desarrollo, para crear un proyecto arquitectónico denominado prototipos de viviendas económicas con tecnologías de paneles. Para tal efecto se planteó un experimento en el que participaron alumnos y profesores.
\end{abstract}

Palabras clave: laboratorio vivo, aprendizaje colaborativo, proyecto arquitectónico, prototipo digital.

\begin{abstract}
The experiment began with the use of a learning environment called living labs that are collaborative learning research environments to involve users in innovation and development, to create an architectural project called affordable housing prototypes with panel technologies, For this purpose, an experiment was proposed in which students and teachers participated. To organize the prototype manufacturing process, a production line was divided into 16 development blocks, and 10 indicators to observe the process, producing results that allow the teaching of architecture to evolve through these new learning environments.

key words: living laboratory, collaborative learning, architectural project, digital prototype.
\end{abstract}

\section{Introducción}

La sostenibilidad ambiental sigue estando ausente en los planes de estudio de la arquitectura a nivel universitario (Dabaieh et al., 2018). En el caso de la Facultad de Arquitectura de la Universidad de Guayaquil, Ecuador, no se menciona al eje transversal relativo a la sustentabilidad, declarados en los objetivos de desarrollo sostenible (ODS), sus objetivos se focalizan en la Arquitectura. Esto puede conducir a la formación de nuevas generaciones de arquitectos que no tengan los conocimientos necesarios para alcanzar objetivos de sostenibilidad global (Alireza Mahdizadeh Hakak et al., 2014).

Este artículo discute una experiencia de enseñanza en un laboratorio vivo, un ecosistema de innovación abierta centrado en el usuario, donde los estudiantes aprenden e intercambian conocimientos de forma colaborativa, que integra procesos concurrentes de investigación e innovación; investiga los roles del aprendizaje a través del

\footnotetext{
${ }_{1}^{1}$ Docente Universidad de Guayaquil. alfredo.carabajoa@ug.edu.ec Orcid 0000-0003-3314-9912

2 Docente Universidad de Guayaquil. .rommy.torres@ug.edu.ec. Orcid 000-0002-9434-3105

${ }_{3}^{3}$ Docente Universidad de Guayaquil. brick.reyesp@ug.edu.ec. Orcid 0000-0001-9456-5034
} 
hacer y la experimentación práctica en la construcción, para arraigar una mayor comprensión de la sostenibilidad en la educación de la arquitectura (formación de los futuros arquitectos). El estudio del diseño es el centro de este documento que se centró en la optimización de recursos para alcanzar una vivienda que utilice la menor cantidad de material, que se construya en el menor tiempo posible, de bajo costo, energéticamente eficiente, adecuada para un clima cálido y árido (como las de los sectores costeros del Ecuador).

Se les pidió a los estudiantes que diseñaran un prototipo de casa que fuera rentable y eficiente en tiempo, que también presentará el menor impacto en el medio ambiente, después de una posible demolición.

El proceso de enseñanza en el living lab también incluyó, como invitados, a arquitectos especialistas en materiales y vivienda de bajo costo. El trabajo se desarrolló en un taller práctico para técnicas de construcción de baja tecnología como preludio al diseño y construcción de un modelo físico a gran escala.

Este documento describe el proceso de laboratorio vivo experimental pedagógico del estudio de diseño y los proyectos resultantes de los estudiantes de la Facultad de Arquitectura de la Universidad de Guayaquil. También muestra las diversas habilidades que los estudiantes adquirieron y sugiere cómo éste tipo de pedagogía se puede transformar en un modelo piloto para la educación en arquitectura verde.

Una serie de desarrollos recientes en educación superior, en especial en campos emergentes de conocimiento, así como las realidades sociales y profesionales cambiantes, dan relevancia a la investigación sobre Living Labs (Agudo, 2017a) en Arquitectura, como nuevas herramientas para una educación holística para el desarrollo sostenible con un enfoque específico en energías renovables y edificios de energía casi nula (Anasagasti, 1995).

Es una responsabilidad social de la universidad promover investigaciones y desarrollos de soluciones innovadoras para conflictos ambientales, sociales y económicos y debe conducir a nuevos procesos de enseñanza, investigación y transferencia de tecnología basados en una fuerte interacción con sus comunidades locales y regionales (Arteaga et al., 2017). Este documento destaca la importancia de Living Labs como infraestructuras de investigación en la educación superior y presenta la futura experiencia educativa específica dentro del marco institucional de la Universidad de Guayaquil, con una investigación en acción sobre educación en sostenibilidadbasada en la experiencia pluridisciplinaria del concurso del Banco Mundial denominado "RESILIENT HOMES CHALLENGE", Architectura Competition World (Espinoza et al., 2018a)., cuya metodología se centraba en trabajo colaborativo para alcanzar varios criterios de evaluación: Resistencia (25\%), Diseño (20\%), Rentabilidad (15\%), Construcción (15\%), Sostenibilidad (15\%) y Presentación (10\%). Precisamente el concurso presentó las condiciones exigentes para poner a prueba dos cosas que se relacionaban con el experimento; lograr una propuesta sostenible de bajo costo y desarrollada en un ambiente colaborativo.

\subsection{Living labs o laboratorios vivos en el aprendizaje de la arquitectura}

La educación en las escuelas de arquitectura debe atender la demanda de un nuevo perfil para los arquitectos, integrando y fomentando las competencias correspondientes a través de nuevas metodologías, herramientas y conceptos de aprendizaje (Encinas et al., 2018; Muy et al., 2019).

Eriksson et al. (2006) propusieron una serie de desarrollos recientes que dan relevancia a la investigación sobre Living Labs en Arquitectura como nuevas herramientas para una educación holística para el desarrollo sostenible con un enfoque específico en las energías renovables, en la vivienda y nuestra vida cotidiana:

a) Una nueva realidad educativa, con redefinición de objetivos en la educación superior, basados en el aprendizaje por competencias y un enfoque más transdisciplinario.

b) Una nueva realidad del conocimiento, con una necesidad urgente de adquirir nuevos conocimientos sobre temas específicos, tales como, edificios de baja energía, materiales de construcción de bajo 
impacto y tecnologías energéticamente eficientes.

c) Una nueva realidad social, cambio hacia la participación, innovaciones sociales constantes y una mayor demanda de estándares ambientales.

d) Una nueva realidad profesional, que requiere más interdisciplinariedad, la capacidad de lidiar con la complejidad y la incertidumbre y nuevas formas de procesos de desarrollo de proyectos basados en la colaboración.

Una fortaleza especial de estos tipos de living labs de arquitectura es su relación inmediata con la vida y el trabajo de las personas, con un enfoque en la vivienda y en el estilo de vida (Eriksson et al., 2006). Esto abre importantes oportunidades para innovar con los usuarios en campos relacionados con la sostenibilidad, como las innovaciones tecnológicas relacionadas con el hogar, el consumo sostenible y los modelos de cambio de estilo de vida. (Cervantes et al., 2019).

Los Living Labs pueden variar desde una difusión de conocimiento a pequeña escala y hogares de experiencia hasta una plataforma de la ciudad para la innovación social con respeto a la sostenibilidad, o la propia ciudad con sus edificios y habitantes como ecosistema de apoyo para la innovación centrada en el usuario, en entornos de la vida real (Castañeda, 2017a). El concepto de Living Lab es el de una plataforma de actividades con un enfoque participativo para la enseñanza y el aprendizaje basado en un prototipo.

Las actividades de enseñanza y aprendizaje tienen lugar paralelamente a proyectos de investigación y actividades de divulgación para crear sinergias entre sectores, personas y disciplinas. Finalmente, Living Lab también es un lugar para la creación de redes de personas con el fin de participar en nuevos proyectos e iniciativas de sostenibilidad, fomentando al factor humano detrás de estas actividades.

La formación del futuro arquitecto, exige nuevas formas de enseñanza que incluya a la producción y construcción colectiva del conocimiento y a los recursos educativos que entran en juego. La investigación de este estudio busca, además, poner de manifiesto la utilidad de los living lab como una estrategia didáctica para las nuevas generaciones de arquitectos.

\section{Metodología}

El concepto de Living Lab está influenciado por la ides de participación del usuario en la investigación y la innovación (Ortiz \& Sanchez, 2017), así como con la creación conjunta y la innovación abierta en entornos de la vida real a través de un enfoque de múltiples actores interesados (academia, empresas y entidades de investigación, pero también la administración local), entendiendo a los estudiantes, investigadores y ciudadanos como los "usuarios" de esta plataforma recién generada (Masseck, 2017).

Otro concepto clave que se utilizó para el desarrollo y fabricación del prototipo de vivienda fue el de LEGOS P, que consiste en piezas que se arman hasta formar un prototipo. Para este fin revisamos algunos procedimientos desarrollados por el profesor de investigación del aprendizaje LEGO papert, Mitchel Resnick en el MIT Media Lab (Resnick, M., and Ocko, S.,1991).

Una vez que la fabricación del prototipo en el living lab haya alcanzado los estándares de eficiencia, ahorro, economía, sustentabilidad, se podrían realizar otras gestiones con las instituciones involucradas como el Ministerio de la Vivienda para la construcción del prototipo real.

Para el proyecto experimental living lab, se utilizó un procedimiento reglamentario de la universidad de Guayaquil, denominado prácticas preprofesionales, que permitió a los estudiantes utilizar 240 horas de prácticas para el desarrollo del proyecto de investigación. La finalidad del living lab, era proponer un sistema de formación para la producción colectiva de un prototipo de vivienda con tecnología de paneles (Espinoza et al., 2018b). 
Para tal efecto, inicialmente se instruyó a los 32 estudiantes que participaron en el proyecto experimental en la utilización de los componentes de los estándares LEED; La certificación LEED (Leadership in Energy and Environmental Design o Liderazgo en Diseño Ambiental y Energético) que utiliza una metodologìa estandarizada de 6 parámetros, con el propósito de aprovechar de una mejor manera el entorno para beneficiar tanto al proyecto arquitectónico como al contexto urbano.

El trabajo propone recomendaciones para optimizar el usos de los recursos, agua, energìa y materiales; también proponen conceptos generales para el manejo y cuidado del paisaje del sitio del proyecto, respetando los recursos naturales del lugar. Sitios Sustentables (24 puntos), Eficiencia en el Uso del Agua (11 puntos), Energía y Atmósfera (33 puntos), Materiales y Recursos (13 puntos), Calidad del Ambiente Interior (19 puntos), Innovación en el diseño (6 puntos).

Posteriormente, el equipo de profesores del proyecto de investigación incluyó y en la propuesta los estándares exigidos por el concurso del Banco Mundial, Resiliencia (25\%), Diseño (20\%), Rentabilidad (15\%), Construcción (15\%), Sostenibilidad (15\%), y Presentación (10\%)(Castañeda, 2017b).

La supervisión del avance del proyecto en el laboratorio estuvo a cargo de 6 profesores que monitoreaban los procesos y resultados. Para el efecto, y sobre la base de los estandares LEED, y los estándares exigidos por el concurso del Banco Mundial, se estructuró un sistema de 10 indicadores que permitía medir el avance del proyecto y 16 bloques de desarrollo del mismo. Los indicadores que se plantearon fueron; 1.-Desarrollo de algoritmos, 2.-Resolución de problemas y grado de innovación, 3.-Planificación y previsión de tareas entre docentes y estudiantes, 4.-Grado de coordinación e interdependencia de los componentes, 5.-Utilización de herramientas tecnológicas, 6.-Grado de ajuste, 7.-Focalizador del atractor, 8.-Grado de velocidad en la investigación de campo, 9.-Defensa de las ideas y grado de desempeño, 10.-Desarrollo de una plataforma colaborativa o registro (Banco Mundial, 2019).

Como todo prototipo de fabricación, se dividió el proyecto en 16 partes a las que llamamos bloques de desarrollo, constituidos por: 1.-Normas de colores, 2.-Kit embalaje, 3.-Manual de construcción, 4.-Instalaciones, 5.-Resinas

y pegantes, 6.-Economía de materiales, 7.-Memoria técnica, 8.-energía y atmosfera, 9.-ubicación y análisis de sitio, 10.-materiales y recursos, 11.- Eficiencia energética, 12.-Calidad de ambiente interior, 13.-Calidad y servicio, 14.-Parámetros de confort, 15.-Aspecto socio-económico-cultural, 16.-Fabricación del prototipo(Marshall et al., 2009). Para complementar esta tarea se realizó un seguimiento muy específico sobre el grado de dominio del software que los estudiantes utilizaron para el desarrollo del proyecto como se explica en el documento de Agudo (2017).

\section{Resultados}

Basándose en la certificación LEED "Leadership in Energy and Environmental Design", se logró formular el prototipo digital de vivienda económica con tecnología de paneles a un estado de optimización superior al de todos los prototipos anteriores experimentados en el living lab, logrando así un estándar aceptable de finalista en el concurso "Resilient Homes Desing Challenge". El diseño de la vivienda de 50,40 m2 de construcción dentro de un terreno de 9 metros de frente por 14 de profundidad, posee la Cocina-Comedor y dormitorios. El experimento logro reducir el número de paneles a 7, y se adecuó el ambiente interior, la edificabilidad y sobre todo la sustentabilidad.

El prototipo tiene un costo estimado de \$9.267, y se lo podrá armar en 72 horas con una duración estimada de 50 años. Para ello se diseñaron básicamente dos tipos de paneles, estructurales y de pared para la elaboración del prototipo, que fueron presentados en el concurso del Banco Mundial. Ver figura 1 
Dos piezas claves en la organización del living lab, fue la división de bloques de tareas para alcanzar el mayor rendimiento, y el monitoreo de los indicadores, con la incorporación de las variables de sostenibilidad para la optimización de los recursos en los materiales, en el tiempo y en la organización, siguiendo los estándares de la línea Ford.

La división de los bloques corresponden al manejo de los estándares de diseño que deben implementar los arquitectos, relativo a las normas de diseño, fabricación, economía, eficiencia ambiental, aspectos culturales, construcción, instalaciones, acabados, transporte y embalaje.

En el trabajo y producción de los bloques se encontró mucha diversidad, a pesar que se dio libertad a los estudiantes para que escojan los bloques de acuerdo a sus fortalezas, se encontraron resultados muy heterogéneos. No obstante el eco sistema debería ser jerarquizado. Esto significa que existen varios niveles de subsistemas de los grupos estudiantiles participantes, encontrándose tres clasificaciones bien estructuradas, grupo de estudiantes desarrolladores, aquellos que realizaban exclusivamente la parte de su trabajo, estudiantes organizadores que enlazaban los productos de los diferentes bloques, y los estudiantes que integraban todo en la plataforma digital. En ese sentido se ubicaron estudiantes coordinadores debido a su mayor fortaleza, el grupo de "las estudiantes lideró la planificación", mientras que los varones lideraron ciertos componentes, y que gradualmente tenían prioridad en la toma de decisiones. Un sistema de este tipo fue fácil de controlar, sin embargo, en muchas ocasiones la rigidez fue grande, y las soluciones vinieron también de los mandos medios. En realidad, no hay jerarquía absoluta, ciertas relaciones cambiaban según las circunstancias. Los circuitos de retroalimentación fueron muy numerosos. Se puede decir que el sistema living lab adoptado tuvo una mutación constante, pero no por ello se perdió el interés de la búsqueda.

Las prácticas profesionales, requerimiento reglamentario de la Universidad de Guayaquil, para los estudiantes, quienes deben destinar 240 horas a proyectos de práctica, en este caso, tributan a la investigación, jugaron un papel importante. Constituyeron un indicador binario porque permitió medir el grado de eficacia y participación de los alumnos en el proyecto y en los bloques, además permitió medir la participación colectiva en la resolución de los problemas. Para ello se utilizaron los informes o actas de cumplimento de metas y tareas grupales e individuales, concluyéndose que las tareas fueron cumplidas $80 \%$.

El aprendizaje que se logró alcanzó el 80\%, fundamentalmente, por los conocimientos simultáneos, en todos los campos mencionados en la metodología expuesta.

El número de foros especializados organizados fueron 12, participaron 32 estudiantes, en 5 campos bien acotados, que fueron los siguientes: campo tecnológico, campo materiales, campo ecológico, campo de vivienda, campo de economía de recursos. También se concretaron 8 foros interdisciplinarios durante el proyecto.

La concepción Legos para la fabricación del prototipo sirvió indiscutiblemente en la experimentación y exploración de paneles, permitió avanzar en el ensamblado y diseño de los paneles que bajo el sistema de autosoporte y autopartes, configuran el prototipo de un sistema constructivo, que dicho sea de paso, está en solicitud de patente, encierra varios pasos; proceso de encaje de los paneles en la malla, las paredes exteriores, las paredes interiores, el tratamiento de las esquinas, las instalaciones centralizadas, tratamiento del piso, tratamiento del techo y el tratamiento de los zócalos para ventanas y puertas.

Respecto a la fabricación del prototipo de vivienda con tecnología de paneles se tomo como premisa fundamental para ahorrar material, utilizar el menor numero de tipo de paneles para abaratar los costos. Se tuvieron dificultades para cuadrar y cerrar los perímetros geométricos de las plantas arquitectónicas del prototipo digital a tal punto que se debió utilizar la figura de un comodín para cerrar los perímetros, pero de una manera muy artesanal dibujando cada panel y sorteando los obstáculos. 
Figura 1

Prototipo presentado por la Universidad de

Guayaquil en el Concurso Banco Mundial

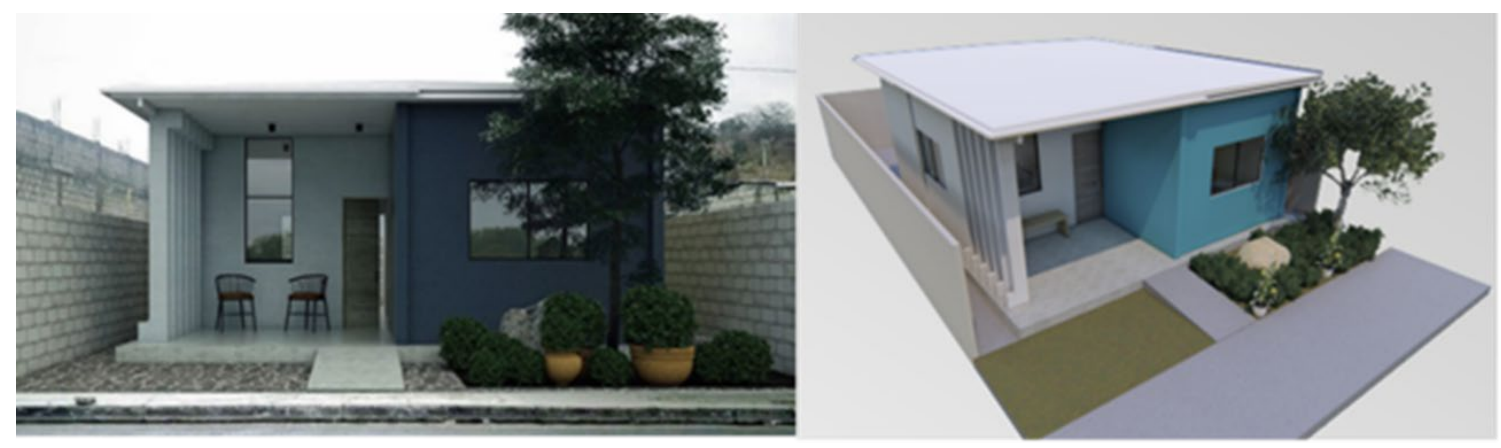

Fuente: Living labs LEGOS P

Esta dificultad hizo que mucho después del concurso, se encontrara uno de los más importantes hallazgos operativos de la investigación geométrica, la utilización de una malla o carril básico que permita ubicar y correr los paneles o vagones, dentro de los carriles, lo que facilitaba enormemente el cerramiento de los perímetros geométricos, y para calcular y dimensionar los tipos y la cantidad exacta para su cierre dependiendo de las demandas de espacio. Ver figura 2.

La investigación y desarrollo del prototipo permitió encontrar 5 tipos de paneles para configurar el prototipo.

Figura 2

Primer prototipo de paneles

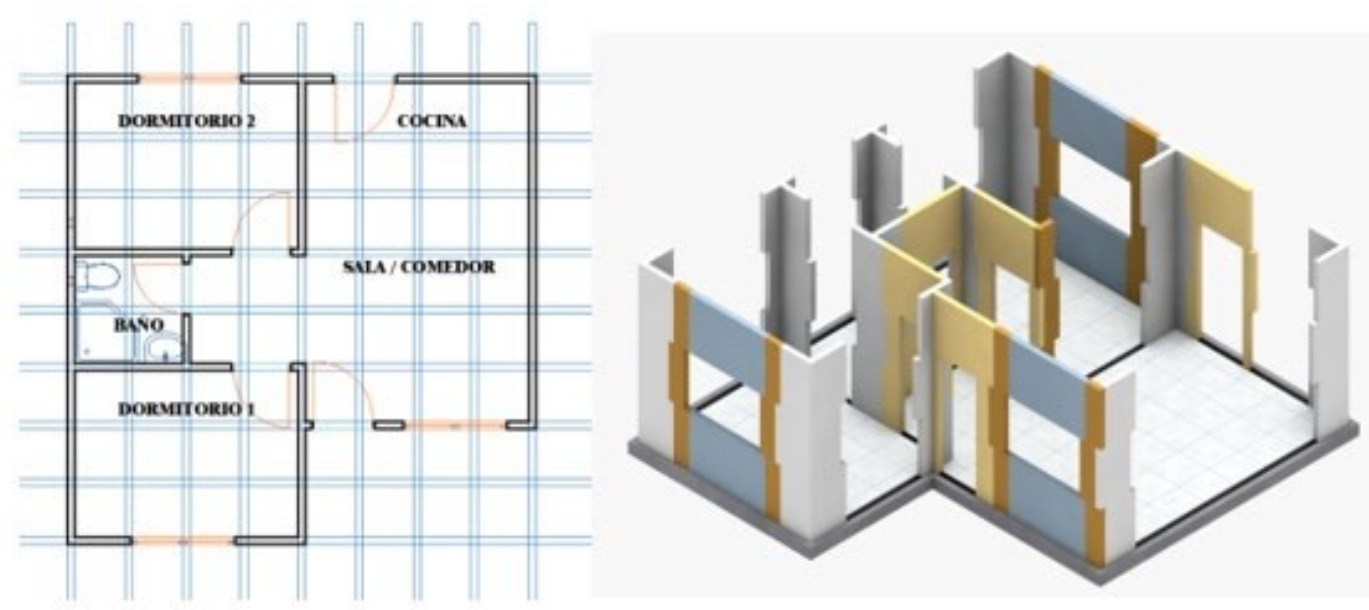

Fuente: Living labs LEGOS P

El procedimiento detallado de exploración de paneles y su volumetría, encierra varios pasos (Agudo, 2017):

\subsection{Proceso de encaje de los paneles exteriores en la malla}

En primer lugar se estableció el dimensionamiento del bloque base, por ejemplo 6 x10 m. Con esa medida se analizó la conveniencia del tamaño y números de los paneles. Estos debían encajar perfectamente tanto por el lado ancho, como por el largo, sin embargo uno de los dos lados debería considerar el número de paneles más el espesor de los paneles del otro lado. Éste fue un gran problema al principio, ya que después de elaborar la maqueta, era necesario llevarlo al plano digital, y al no considerar esta situación particular no cerraba el polígono de la planta. Esto sin duda alguna, obligaría a introducir un panel con una dimensión distinta en su ancho. 
Por lo general la planta se desarrolla bajo el principio de Loft. Como las dimensiones del área neta construida es pequeña (entre 40 y $50 \mathrm{~m} 2$ ), fue necesario considerar un gran espacio con pocas divisiones para que los usuarios puedan tener una libertad para organizar el espacio de acuerdo a sus patrones culturales. Su emplazamiento se estima en un terreno entre 100 y $120 \mathrm{~m} 2$.

\subsection{El piso}

El proyecto propone que los paneles encajen en una ranura del piso para darle estabilidad y firmeza, para ello se requerirá que los canales sean más largos en las intersecciones para poder mover la panelería hasta ajustarlos.

\subsection{Proceso de tratamiento de las esquinas}

Los paneles necesitan tener un diseño especial en las esquinas porque deben ser resistentes, ya que el sistema que se ha implementado es de "Autosoporte". Por lo tanto, las esquinas juegan un importante papel ya que sostienen toda la panelería de ambos lados. Para tal efecto, inicialmente se consideró el sistema machihembrado o machimbrado, término usado en la industria de la madera para unir piezas, donde la protuberancia encaja perfectamente en una ranura pero carecía de las condiciones para sostener una esquina, por lo que se escogió de la familia de los entramados con forma de dentado simple que son las más resistentes.

\subsection{Paredes interiores}

Las paredes medianeras necesitan interceptarse con las paredes perimetrales, formando las " $T$ ". Para el efecto, no se requieren encajes fuertes, bastaría con un empotramiento que consiste en que el panel de la pared interior encaje en la pared exterior a través de una protuberancia dentada, y que la pared exterior contenga una ranura.

\subsection{Instalaciones}

Para economizar, las instalaciones deben estar centralizadas en un solo sitio, de tal forma que la red de agua potable y servida, para cocina y baño esté cerca y puedan superponerse o empotrarse en la panelería.

\subsection{El techo}

Al igual que el piso, el proyecto propone que los paneles de las paredes encajen en las ranuras de los paneles de techo para darle estabilidad y firmeza, para ello será necesario conocer con mucha precisión el dimensionamiento del entramado de las paredes. El techo será más firme si se apoya en las modulaciones de las paredes cerradas de los baños.

\subsection{El tratamiento de zócalos para ventanas y puertas}

La ubicación de puertas y ventanas requerirá una destreza de manejo de los paneles, los zócalos deberán ser soportados por el diseño de una panelería dentada. La experimentación de ensamblado y diseño de los paneles permitieron avanzar en el prototipo que se presentó en el concurso del Banco Mundial. Ver figura 3.

Debe destacarse que el equipo de diseño estudiantil miró más allá de los diseños de casas prefabricadas, permitiendo incorporar técnicas de construcción y materiales locales en sus diseños, con un objetivo dirigido hacia la facilidad de construcción.

La fabricación del prototipo debería estar diseñado a partir de estructuras como un kit de piezas a partir de un modelo 3D. Usamos una variedad de métodos de fabricación de aditivo a sustractivo. La idea central era buscar el armado mas adecuado, sencillo y practico.

El marco teórico de nuestro sistema se basa en las técnicas de modelización generativa encontradas en el diseño evolutivo. El sistema, guiado por reglas, descompone una forma 3D inicial en partes de enclavamiento más 
pequeñas preparadas para la fabricación y montaje como un kit físico de piezas. En teoría, nuestra gramática evolutiva puede apoyar la entrega de cualquier edificio de tamaño y cualquier número de componentes. El objetivo es ayudar a las personas a que armen sus casas con facilidad en 72 horas, tal como se presentó la propuesta al concurso del Banco Mundial.

Figura 3

El segundo prototipo

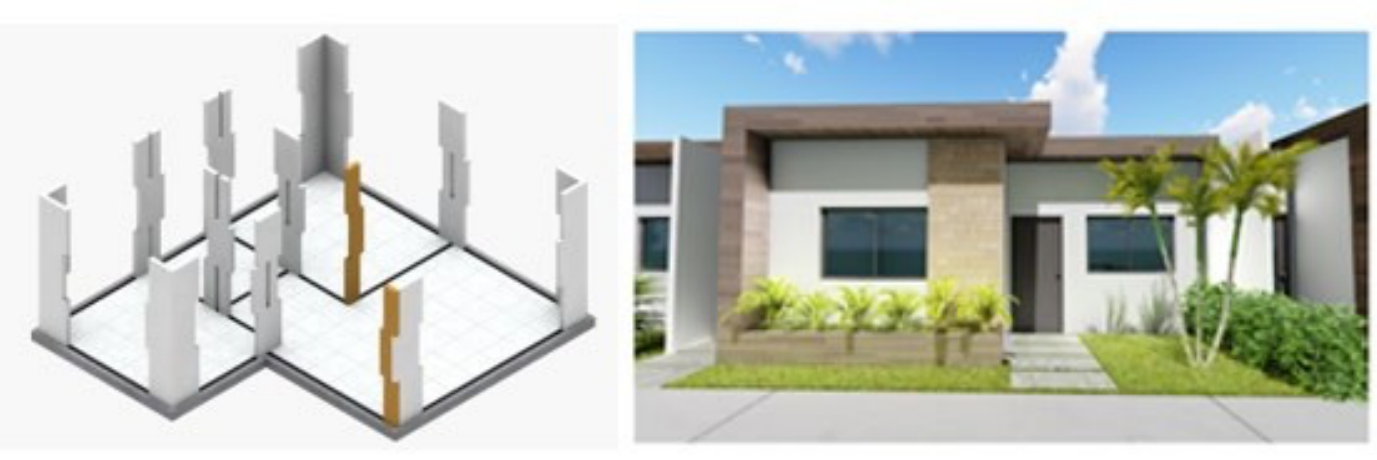

Fuente: Living labs LEGOS P

Respecto al grado de dominio de las herramientas digitales que presentaron los estudiantes para el desarrollo del Prototipo de Vivienda Económica de la Facultad de Arquitectura de la Universidad de Guayaquil, se puede concluir que los resultados son alarmantes, en general el dominio de softwars utilizados resultaron muy básicos, siendo autocad el de mayor uso, skechtup medianamente, y los de postproducción como ilustrator, fotoshop, muy básicos. En tanto que archicad que es un software más versátil, y tiene un mayor impacto en la calidad de la producción arquitectónica, resultaba muy incipiente, esto indudablemente afecta en la calidad de la representación espacial del proyecto, restando competitividad al estudiante.

En suma, las observaciones realizadas en el proceso living lab, fortalecen la idea de un modelo de enseñanza colaborativo que enfatiza la construcción social del conocimiento, siendo clave la combinación y énfasis de desarrollo de los bloques. Esta combinación nos permite efectuar las siguientes predicciones; el grupo alcanzó un nivel alto de dominio de práctica experimental utilizando dos ejercicios de estándares; la fabricación digital del prototipo estándar LEED, y los estándares propuestos por el concurso del Banco Mundial denominado Resilient Homes Design Challenge (2018). Y que precisamente la experimentación de los bloques de desarrollo permitió ubicar al grupo Legos $\mathrm{P}$ como finalista, dicha práctica permitió avanzar rápido en los objetivos propuestos para el concurso (en 2 semanas), pero no por ello se alcanzó precisión tecnológica.

Una segunda predicción clave es que las herramientas tecnológicas jugaron un papel importante en el desarrollo del prototipo (Torres et al., 2018), ya que integra todos los bloques de desarrollo, sin embargo, no tuvieron un papel de alto desarrollo. En forma concomitante se evidenció pocos escenarios para la búsqueda de una nueva geometría arquitectónica, acentuada por una básica formación, a pesar de que los elementos constructivos correspondieron eficazmente al desarrollo de los prototipos.

\section{Conclusiones}

Implementar en el living lab, una propuesta didáctica para la fabricación del prototipo de vivienda económica con tecnología de paneles fundamentada en la enseñanza por descubrimientos (recurso experimental), requiere además del diseño de los prototipos y experimentos, de una serie de guías de instrucción de apoyo al docente y al alumno para armonizar la parte cognocitiva del aprendizaje programado, con el trabajo experimental del prototipo. 
Para el efecto los docentes y estudiantes del proyecto deberían seguir unos lineamientos de desarrollo del proyecto de investigación, en las áreas de tecnología de la representación del espacio, sostenibilidad y optimización de recursos, materiales, y economía y producción en masas basándose los principios de Tylor y Ford.

En ese marco, las guías y foros establecidos deberían ser discutidos, tales como la certificación LEED "Leadership in Energy and Environmental Design”, las guías establecidas por el concurso del Banco Mundial, las guías LEGO Papert de Investigación de Aprendizaje en el MIT Media Lab, las guías Tylor y Ford, las guías de la arquitectura de MAKOTO MASUZAWA, las guías MIUS, Technology Assessment of Modular Integrated Utility Systems, las guías de Utilizacion Optimización de recursos in situ (In-Situ Resource Utilization -“ISRU”), y las guías sobre los materiales de poliuretano expandido y pulioretano. Las guías permitieron alimentar el trabajo experimental y finalmente sintetizar y formular una propia guía que permita entregarla al usuario para que arme su casa. Esto ya establecía una gran diferencia como se maneja la asignatura de proyectos en la Facultad de Arquitectura.

Los resultados obtenidos con las guías de construcción, discutidas, validadas y probadas en el laboratorio living lab LEGOS $\mathrm{P}$, demostraron ser una herramienta eficaz para el logro de la didáctica experimental, muchas veces poco implementada en el desarrollo y experimento del proyecto arquitectónico en el aula tradicional de proyectos.

Uno de los resultados más destacados en el living lab, de fabricación del prototipo, era pasar de un foro a otro, de una guía a otra, lo que permitió la construcción de por lo menos 9 prototipos experimentales que paulatinamente se iban mejorando en la medida que los foros y las guías de instrucción se discutían. Los procesos de mejoras siempre atendían el objetivo del trabajo que consistía en desarrollar una vivienda económica, con el menor número de tipos de paneles para construirlo en 72 horas.

Eso era posible porque, de forma complementaria, el proceso de propuesta didáctica debería tener una visión distinta, esto es descomponer el problema en sus partes y para ello se implementaron 16 bloques de tareas para desarrollar el prototipo, con un sistema de indicadores. Esto ha representado un salto importante en la forma que se enseña tradicionalmente Taller de proyectos en sus tres etapas (investigación, programación y diseño), puesto que la mecánica de la propuesta de bloques incluía el desarrollo de estas etapas.

La implementación del living lab permitió, exitosamente, la construcción colectiva del conocimiento para fabricar el prototipo de vivienda y desde luego, ayudo a formular eficazmente la propuesta para el concurso del Banco Mundial, pudiendo monitorear los acontecimientos del ecosistema de bloques y sus partes constitutivas. La división de Bloques permitirá desarrollar en un futuro cercano métodos matemáticos informatizados para realizar ajustes automáticos en los bloques para alcanzar los resultados más eficientes. Este planteamiento formativo tendrá enorme impacto en la formación del arquitecto, especialmente en las aulas de aprendizaje de las universidades, permitiendo el desarrollo del conocimiento colectivo a menor coste, con respuestas rápidas aprovechando las fortalezas sistémicas de los actores.

Debe indicarse que el concepto del diseño del laboratorio distaba del concepto del aula del taller de proyectos, ya que el living estaba constituido por 32 alumnos y 6 docentes y los conocimientos de las diferentes disciplinas se encontraban en el mismo laboratorio, los alumnos no tenían que salir del laboratorio para recibir guías complementarias en otra aula de clases.

No menos importante fue la participación de los estudiantes en el proyecto Legos $P$, bajo la modalidad de horas de prácticas pre profesionales tributadas a investigación, sin embargo, esta normativa fue cambiada de forma inesperada con otros requisitos de notas para los estudiantes, lo cual no permitió que el proyecto pudiera seguir experimentando. 
Las medidas propuestas aquí, conducen necesariamente a buscar las reformas de la Ley Orgánica de Educación Superior (LOES) de Ecuador, y al Reglamento de Régimen Académico para formular y establecer nuevos entornos de aprendizaje que permitan una formación más rápida y eficaz para resolver problemas.

\section{Referencias bibliográficas}

Agudo, J. (2017a). Sostenibilidad y fabrication laboratory aplicado a la arquitectura: Neil A. Gershenfeld y el How to Make (Almost)Anything. :

https://www.researchgate.net/publication/331233695_Sostenibilidad_y_Fabrication_Laboratory_aplicad o_a_la_Arquitectura_Neil_A_Gershenfeld_y_el_How_to_Make_Almost_Anything

Alireza Mahdizadeh Hakak, Nimish Biloria, \& Armaghan Ahmadi Venhari. (2014). Creativity in architecture-A Review on Effective Parameters Correlated with Creativity in Architectural, Design. Journal of Civil Engineering and Architecture, 8(11).

http://www.davidpublisher.org/index.php/Home/Article/index?id=7651.html

Anasagasti, T. de. (1995). +enseñanza de la arquitectura: Cultura moderna técnico artística. https://www.coam.org/media/Default\%20Files/fundacion/biblioteca/revista-arquitectura-100/19811986/docs/revista-articulos/revista-arquitectura-1983-n240-pag35-36.pdf

Arteaga, G., Escobar, D., \& Galindo, J. (2017). Efectos colaterales en la superficie urbana por aplicación de decreto sobre espacio público en colombia. Revista Espacios, 38(07)2017 p. 07. https://www.revistaespacios.com/a17v38n07/17380707.html

Banco Mundial. (2019). Winners of «resilient homes design challenge» announced: https://www.worldbank.org/en/topic/disasterriskmanagement/brief/resilient-homes-challenge. World Bank, (2019). Resilient homes design challenge. Washington, DC: World Bank.: http://viewer.zmags.com/publication/858e96ae\#/858e96ae/143

Castañeda, C. A. T. (2017). Construcciones sostenibles y certificaciones leed en colombia. 110:https://repository.uamerica.edu.co/bitstream/20.500.11839/7034/1/465230-2017-I-GA.pdf

Cervantes, S. R. S., Ordoñez, F., \& Santistevan, S. (2019). Vivienda social: prototipo de vivienda económica con tecnología de paneles prefabricados para la ciudad de Guayaquil. Universidad de Guayaquil. http://repositorio.ug.edu.ec/handle/redug/39767

Dabaieh, M., El-Mahdy, D., \& Maguid, D. (2018). Living labs as a pedagogical teaching tool for green building design and construction in hot arid regions. International Journal of Architectural Research: ArchNet-IJAR, $12,338$.

https://www.researchgate.net/publication/324099103_Living_labs_as_a_pedagogical_teaching_tool_for_ green_building_design_and_construction_in_hotarid_regions

Encinas, K. L. P., Díaz, Y. E., \& Chacón, M. L. L. (2018). Calidad en los procesos educativos mediados por tecnología. Revista Espacios, 39(30)2018 p. 06. https://www.revistaespacios.com/a18v39n30/18393006.html

Eriksson, M., Niitamo, V.-P., Kulkki, S., \& Hribernik, K. A. (2006). Living labs as a multi-contextual r\&d methodology. 2006 ieee International Technology Management Conference (ICE), 1- 8. https://doi.org/10.1109/ICE.2006.7477082

Espinoza, F., Reyes, L., Carbajo, A., Torres, R., \& Rendón, I. (2018b). THE fundamental algorithms that support the spatial geometry for the development of the technology of " "p-legos"”" panels applicable to affordable 
housing». /issues/fundamental-algorithms-support-spatial- geometry-development-technology-p-legospanels.

http://webcache.googleusercontent.com/search?q=cache:Icaphtkgjlyj:journalijcir.com/archive/201809\&hl $=e s \& g \mid=e c \& s t r i p=1 \& v w s r c=0$ :

Marín, S. L., Gallego, J. A. L., Maldonado, J. J. C., \& Murillo, A. P. (2018). Guía interactiva para el desarrollo de proyectos bajo la metodología de marco lógico. Revista Espacios, 39(47)2018 p. 25.

http://www.revistaespacios.com/a18v39n47/a18v39n47p25.pdf

Marshall, W. C., Girón, A., Correa, E., Rodríguez, P., Cabrera Morales, S., Vidal, G., \& Martínez, I. (2009). John Kenneth Galbraith: homenaje en el centenario de su natalicio. Ola financiera, 2(2). https://doi.org/10.22201/fe.18701442e.2009.2.23039

Masseck, T. (2017). Living Labs in Architecture as Innovation Arenas within Higher Education Institutions. Energy Procedia, 115, 383-389. https://doi.org/10.1016/j.egypro.2017.05.035

Muy, N. I., Moreta, J. L., Amancha, M. F., \& Acosta, I. E. (2019). Propuesta metodológica para determinar el valor patrimonial de viviendas históricas en sectores urbanos. Caso de estudio. Revista EspacioS, 40(13)2019 p. 15. https://www.revistaespacios.com/a19v40n13/19401315.html

Niitamo, V.-P.; Kulkki, S.; Eriksson, M.; Hribernik, K. A.: state-of-the-art and good practice in the field of living labs, Proceedings of the 12th International Conference on Concurrent Enterprising: Innovative Products and Services through Collaborative Networks, Milan, Italy, 2006, 349-357.

www.researchgate.net/publication/272566802_LIVING_LABS_FOR_INNOVATION_AND_DEVELOPMENT_O F_INFORMATION_AND_COMMUNICATION_TECHNOLOGY_A_LITERATURE_REVIEW

Ortiz, M., \& Sanchez, B. (2017). Propuesta de una metodología para la gestión de proyectos de infraestructura y socio productivos en una gerencia de desarrollo social. Revista Espacios, 38(21)2017 p. 24. http://w.revistaespacios.com/a17v38n21/17382124.html

Resnick, M., and Ocko, S. (1991). LEGO/Logo: Learning Through and About Design. In Constructionism, edited by I. Harel \& S. Papert. Ablex Publishing. https://llk.media.mit.edu/papers/II.html

Torres, S. A. C., Ramos, J. B. V., \& Valbuena, S. J. (2018). Entorno de aprendizaje virtual adaptativo para la gestión de la evaluación. Revista Espacios, 39(20)2018 p.32. http://www.revistaespacios.com/a18v39n20/a18v39n20p32.pdf

Esta obra está bajo una Licencia Creative Commons Attribución-NoCommercial 4.0 International

\section{(c) EY-NO}

